\title{
Splenic NKG2D confers resilience versus susceptibility in mice after chronic social defeat stress: beneficial effects of $(R)$-ketamine
}

\author{
Kai Zhang ${ }^{1,3} \cdot$ Akemi Sakamoto $^{2} \cdot$ Lijia Chang $^{1} \cdot$ Youge Qu$^{1} \cdot$ Siming Wang ${ }^{1} \cdot$ Yaoyu Pu ${ }^{1} \cdot$ Yunfei Tan ${ }^{1}$. \\ Xingming Wang ${ }^{1} \cdot$ Yuko Fujita $^{1} \cdot$ Tamaki Ishima $^{1} \cdot$ Masahiko Hatano ${ }^{2} \cdot$ Kenji Hashimoto $^{1}{ }^{1}$
}

Received: 8 November 2019 / Accepted: 17 December 2019 / Published online: 24 December 2019

(c) The Author(s) 2019

\begin{abstract}
The spleen is a large immune organ that plays a key role in the immune system. The precise molecular mechanisms underlying the relationship between the spleen and stress-related psychiatric disorders are unknown. Here we investigated the role of spleen in stress-related psychiatric disorders. FACS analysis was applied to determine the contribution of the spleen to susceptibility and resilience in mice that were subjected to chronic social defeat stress (CSDS). We found a notable increase in splenic volume and weight in CSDS-susceptible mice compared to control (no CSDS) mice and CSDS-resilient mice. The number of granulocytes, but not of T cells and B cells, in the spleen of susceptible mice was higher than in the spleen of both control and resilient mice. Interestingly, NKG2D (natural killer group 2, member D) expression in the spleen of CSDS-susceptible mice was higher than that in control mice and CSDS-resilient mice. In addition, NKG2D expression in the spleen of patients with depression was higher than that in controls. Both increased splenic weight and increased splenic NKG2D expression in CSDS-susceptible mice were ameliorated after a subsequent administration of $(R)$-ketamine. The present findings indicate a novel role of splenic NKG2D in stress susceptibility versus resilience in mice subjected to CSDS. Furthermore, abnormalities in splenic functions in CSDS-susceptible mice were ameliorated after subsequent injection of $(R)$-ketamine. Thus, the brain-spleen axis might, at least in part, contribute to the pathogenesis of stress-related psychiatric disorders such as depression.
\end{abstract}

Keywords Antidepressant $\cdot$ Brain-spleen axis $\cdot(R)$-ketamine $\cdot$ NKG2D $\cdot$ Spleen

\section{Introduction}

Resilience is the ability to adapt successfully to stress and adversity. Stress resilience is mediated by adaptive changes in the central nervous system (CNS) and peripheral immune system, but the precise mechanisms behind stress

Electronic supplementary material The online version of this article (https://doi.org/10.1007/s00406-019-01092-z) contains supplementary material, which is available to authorized users.

Kenji Hashimoto

hashimoto@faculty.chiba-u.jp

1 Division of Clinical Neuroscience, Chiba University Center for Forensic Mental Health, 1-8-1 Inohana, Chiba 260-8670, Japan

2 Department of Biomedical Science, Chiba University Graduate School of Medicine, Chiba 260-8670, Japan

3 Present Address: Department of Psychiatry, Chaohu Hospital of Anhui Medical University, Hefei 238000, China susceptibility and resilience are unknown [1-7]. Mounting evidence indicates that the immune system plays a key role in stress-related psychiatric disorders such as major depressive disorder (MDD) [6-10]. Stress-induced inflammatory events in the CNS and peripheral immune system are linked with both susceptibility and resilience to stress [2, 7, 11]. Along with physiological and transcriptional adaptations of specific brain circuits, a crucial role in stress resilience is suggested for cellular and humoral factors of the immune system and changes at the interface between the brain and the periphery [7].

The spleen is the largest secondary immune organ, and it plays a key role in the development of stress-related psychiatric disorders [12-14]. Through a highly organized lymphoid compartment, the spleen can not only mount complex adaptive immune responses but also remove pathogens effectively from the blood [12]. It has been reported that chronic social defeat stress (CSDS), a rodent model of depression, increases the percentages of splenic $\mathrm{CD} 11 \mathrm{~b}^{+}$myeloid cells, 
granulocytes, and $\mathrm{CD} 11 \mathrm{c}^{+}$dendritic cells and reduces the percentages of splenic natural killer (NK) cells $[15,16]$. A recent study has shown that the spleen has a role in restraint stress-induced changes in the distribution of leukocytes in the blood [17]. Despite the effects of stress on the spleen being well-known, the precise molecular mechanisms underlying abnormalities in splenic functions caused by CSDS are still unknown. Moreover, there has been no report about the role of the spleen in stress susceptibility versus resilience in mice subjected to CSDS.

The aim of this study was to examine whether the spleen is involved in stress-related psychiatric disorders. Initially, we investigated the role of the spleen in stress susceptibility versus resilience in mice subjected to CSDS. Then, we studied the role of splenic NKG2D (Natural Killer Group 2 , member D) in stress susceptibility versus resilience in mice subjected to CSDS, as NKG2D plays a vital role in the immune system [18-21]. Next, we measured NKG2D expression in the parietal cortex and the spleen from postmortem tissues from both controls and patients with major psychiatric disorders, including MDD, schizophrenia (SZ), and bipolar disorder (BD). The $N$-methyl-D-aspartate receptor antagonist $(R, S)$-ketamine is well-known to have robust antidepressant effects in treatment-resistant patients with depression [22-27]. Finally, we investigated whether a more potent enantiomer of $(R, S)$-ketamine, the rapid-acting and sustained antidepressant $(R)$-ketamine [25-34], can ameliorate abnormal splenic functions (i.e., increased volume and splenic functions) in CSDS-susceptible mice.

\section{Methods and materials}

\section{Animals}

Male adult C57BL/6 mice, aged 8 weeks (body weight 20-25 g, Japan SLC, Inc., Hamamatsu, Japan), and male adult CD1 mice, aged 14 weeks (body weight $40-45$ g, Japan SLC, Inc., Hamamatsu, Japan) were used in the experiments. Animals were housed at a controlled temperature and under $12 \mathrm{~h} \mathrm{light/dark} \mathrm{cycles} \mathrm{(lights} \mathrm{on} \mathrm{between} \mathrm{07:00} \mathrm{and} \mathrm{19:00),}$ with food and water ad libitum. The study was approved by the Chiba University Institutional Animal Care and Use Committee.

\section{Compounds and treatment}

$(R)$-ketamine hydrochloride was prepared by recrystallizing $(R, S)$-ketamine and $\mathrm{D}$-(-)-tartaric acid [28]. The dose $(10 \mathrm{mg} / \mathrm{kg}$ as hydrochloride salt) of $(R)$-ketamine was selected as reported previously [29, 32-35]. Other reagents were purchased commercially.

\section{CSDS model}

The CSDS procedure was performed as reported previously [29, 32-36]. Each day, for a total of 10 days, the C57BL/6 mice underwent 10 min exposure to a different $C D 1$ aggressor mouse. At the end of the social defeat session, the resident CD1 mouse and the intruder mouse were housed in one half of the cage and separated by a perforated Plexiglas divider to enable visual, olfactory, and auditory contact for the remainder of the 24-h period. All mice were housed individually at $24 \mathrm{~h}$ after the final session. On day 11 , we conducted a social interaction test (SIT) to identify subgroups of mice that were susceptible to and not susceptible to social defeat stress. This was achieved by placing mice in an interaction test box $(42 \mathrm{~cm} \times 42 \mathrm{~cm})$, with an empty wire-mesh cage $(10 \mathrm{~cm} \times 4.5 \mathrm{~cm})$ situated at one end. We tracked the movement of the mice $2.5 \mathrm{~min}$, followed by a further $2.5 \mathrm{~min}$ in the presence of an unfamiliar aggressor confined in the wire-mesh cage. We used a stopwatch to record the length of time the subject spent in the "interaction zone" (defined as the 8 -cm-wide area surrounding the wire-mesh cage). The interaction ratio was calculated as time spent in an interaction zone with an aggressor/time spent in an interaction zone without an aggressor. An interaction ratio of 1 was set as the cutoff: mice with scores $<1$ were defined as "susceptible" to social defeat stress, whereas mice with scores $\geq 1$ were defined as "resilient". Approximately $70-80 \%$ of the mice were CSDS-susceptible. Susceptible mice were divided randomly in subsequent experiments involving administration of either saline or $(R)$-ketamine. Control mice were housed in the same cage before the behavioral tests.

\section{Antidepressant effects of (R,S)-ketamine and $(R)$-ketamine in a CSDS model}

CSDS-susceptible mice were administered either saline $(10 \mathrm{ml} / \mathrm{kg})$ or $(R)$-ketamine $(10 \mathrm{mg} / \mathrm{kg})$ intraperitoneally (i.p.). Then, behavioral tests, including locomotion, a tail suspension test (TST), a forced swimming test (FST), and a one $\%$ sucrose preference test (SPT) were carried out as reported previously [29, 32-35, 37-39].

Locomotion Locomotor activity was measured using an animal movement analysis system SCANETMV-40 (MELQUEST Co., Ltd., Toyama, Japan). The animals were placed in experimental cages (length $560 \mathrm{~mm} \times$ width $560 \mathrm{~mm} \times$ height $330 \mathrm{~mm}$ ). The cumulative exercise was recorded for $60 \mathrm{~min}$. The ages were cleaned after each testing session.

TST We placed a small piece of adhesive tape approximately $2 \mathrm{~cm}$ from the tip of each mouse's tail. We punched a single hole in the tape and hung the mice individually on 
hooks. Immobility was recorded over $10 \mathrm{~min}$. Mice were regarded as immobile only when they hung passively and were completely motionless.

FST The FST was conducted using an automated forcedswim apparatus SCANETMV-40 (MELQUEST Co., Ltd., Toyama, Japan). The mice were placed individually in cylinders (diameter: $23 \mathrm{~cm}$; height: $31 \mathrm{~cm}$ ) containing $15 \mathrm{~cm}$ of water that was maintained at $23 \pm 1{ }^{\circ} \mathrm{C}$. The analysis software of the apparatus was used to record the immobility time from activity time as (total)-(active) time. The immobility time was recorded over 6 min.

SPT Mice were fed a water and $1 \%$ sucrose solution for $48 \mathrm{~h}$, after which they underwent $4 \mathrm{~h}$ deprivation of water and food and $1 \mathrm{~h}$ exposure to two identical bottles, one containing water, and the other containing a $1 \%$ sucrose solution. These bottles were weighed both before and at the end of this period. The sucrose preference was calculated as the percentage of sucrose solution consumption to the total liquid consumption.

\section{Western blot analysis of NKG2D in mouse samples}

The animals were killed by cervical dislocation and the spleens were removed rapidly. Half of each spleen was used for Western blot analysis and FACS analysis, respectively. The tissues were stored at $-80^{\circ} \mathrm{C}$ prior to use. We performed Western blot analysis as reported previously [29, 34, 38, 39]. Tissue samples were homogenized in Laemmli lysis buffer. Aliquots $(20 \mu \mathrm{g})$ of protein were measured using the DC protein assay kit (Bio-Rad) and incubated at $95^{\circ} \mathrm{C}$ for $5 \mathrm{~min}$, with an equal volume of $125 \mathrm{mM}$ Tris- $\mathrm{HCl}, \mathrm{pH} 6.8 ; 20 \%$ glycerol; $0.1 \%$ bromophenol blue; $10 \% \beta$-mercaptoethanol and $4 \%$ SDS. The proteins were then subjected to SDS polyacrylamide gel electrophoresis using AnyKD minigels (Mini-PROTEAN TGX Precast Gel; Bio-Rad) and transferred onto PVDF membranes using a Trans Blot Mini Cell (Bio-Rad). For immunodetection, the blots were blocked with $2 \%$ BSA in TBST (TBS + 0.1\% Tween-20) at room temperature for $1 \mathrm{~h}$ and kept with anti-NKG2D primary antibodies (1:1,000; Code No. ab203353: Abcam, Tokyo, Japan) at $4{ }^{\circ} \mathrm{C}$ overnight. The following day, blots were washed three times in TBST and incubated at room temperature for $1 \mathrm{~h}$ with horseradish peroxidase-conjugated anti-rabbit antibody $(1: 10,000)$. Following a final three washes with TBST, bands were detected using enhanced chemiluminescence (ECL) along with the Western Blotting Detection system (GE Healthcare Bioscience). The blots were then washed three times in TBST and incubated with the primary antibody directed against $\beta$-actin (1:10,000; Sigma-Aldrich). Images were captured using a Fuji LAS3000-mini imaging system (Fujifilm, Tokyo, Japan), and immunoreactive bands were quantified.

\section{Western blot analysis of NKG2D in human postmortem tissues}

Human postmortem parietal cortex (Brodmann area 7) and spleen from both normal controls $(n=15)$ and MDD $(n=15), \mathrm{SZ}(n=15)$, and $\mathrm{BD}(n=15)$ patients were obtained from the Stanley Foundation Brain Collection (Bethesda, MD) [40-43]. Spleen samples from three SZ patients and one MDD patient were not included [42]. Medical examiners collected the specimens, and permission was obtained from the next of kin in all cases. The demographic, clinical, and storage information for the cases had been published previously [40]. Each diagnostic group was matched based on several parameters, such as age at death, gender, postmortem interval, brain $\mathrm{pH}$, and brain weight. This study was approved by the Research Ethics Committee of the Graduate School of Medicine, Chiba University. Western blot analysis of NKG2D in the parietal cortex and spleen was performed as described above.

\section{FACS analysis}

Mouse spleen tissues were mashed and passed through a $70 \mu \mathrm{m}$ mesh to prepare a single cell suspension and then subjected to FACS analysis. Spleen cells were suspended and their numbers were counted using an automated cell counter (BIO-RAD, Alfred Nobel Drive, CA). We stained $10^{6}$ cells with various monoclonal antibodies against cell surface antigens for $30 \mathrm{~min}$ at $4{ }^{\circ} \mathrm{C}$ and then washed them with an FACS buffer. For staining, we used the following antibodies: anti TCRb-FITC (cat\# 553170: BD Bioscience, Franklin Lakes, NJ), anti-B220-PE (cat\# 553090: BD Bioscience), anti-NKG2D- allophycocyanin (cat\# 17-5882-82: eBioscience), anti-Gr1-PE (cat\# 55161: BD Bioscience), and anti-DX5-PE (cat\# 553,858: BD Bioscience). The stained cells were analyzed using FACSCantII and FlowJo software (BD Bioscience).

\section{Statistical analysis}

The data are shown as the mean \pm standard error of the mean (S.E.M.). Data were analyzed using PASW Statistics 20 (formerly SPSS Statistics; SPSS) and one-way analysis of variance, followed by a post-hoc Tukey test. $P$ values $<0.05$ were considered statistically significant.

\section{Results}

\section{FACS analysis of spleen samples}

Spleen samples were collected from control mice (no CSDS), CSDS-susceptible mice, and CSDS-resilient mice 
(Fig. 1a). Unexpectedly, the spleens in the CSDS-susceptible mice were significantly larger and heavier than those of both control mice and CSDS-resilient mice (Fig. 1b). We used FACS analysis to count the numbers of T cells, B cells, and granulocytes in the spleens from the three groups. The total cell numbers from the spleens of CSDS-susceptible mice were significantly higher than were those from the spleens of both control mice and CSDS-resilient mice (Fig. 1c). Conversely, the numbers of $\mathrm{T}$ cells and $\mathrm{B}$ cells were similar in the three groups (Fig. 1d, e). Further, the numbers of $\mathrm{Gr}^{+}$ granulocytes in the spleens of CSDS-susceptible mice were significantly higher than in both control mice and CSDSresilient mice (Fig. 1f). Interestingly, there were positive correlations between the number of $\mathrm{Gr}^{+}$cells and spleen weight in all groups (Fig. 1g). Collectively, it is probable that the increased spleen weight in CSDS-susceptible mice might be due to the spleens in these animals having a greater number of granulocytes.

\section{Relationship between depression-like phenotypes and splenic NKG2D expression}

After CSDS, CSDS-susceptible mice and CSDS-resilient mice were divided by means of a SIT (Fig. 2a). Then, we conducted behavioral tests, including locomotion, TST, FST, and SPT (Fig. 2a). There were no changes in locomotion in the three groups (Fig. 2b). The immobility time of TST and
FST in the CSDS-susceptible mice was significantly higher than in both control mice and CSDS-resilient mice (Fig. 2c, d). Moreover, in CSDS-susceptible mice the sucrose preference of SPT was significantly lower than was the case in both control mice and CSDS-resilient mice (Fig. 2e).

NKG2D is a lectin-like type 2 transmembrane stimulatory immunoreceptor that is expressed by all NK cells and by subsets of T cells [18-21]. Because of the key contribution of NKG2D in the immune system [18-20,44], we examined the role of NKG2D in the spleen in a CSDS model. Western blot analysis revealed that NKG2D expression in the spleen of CSDS-susceptible mice was significantly higher than that in both control mice and CSDS-resilient mice (Fig. 2f). Interestingly, there were positive correlations between the immobility time of TST (or FST) and NKG2D expression in the spleen in the three groups (Fig. $2 \mathrm{~g}$, h). These findings indicate that splenic NKG2D may play a role in depressionlike phenotypes in a CSDS model.

\section{Expression of NKG2D protein in the postmortem tissues (parietal cortex and spleen) from patients with MDD, SZ, and BD}

We conducted Western blot analysis of NKG2D protein in the parietal cortex and spleen from patients with MDD, SZ, or BD. There were no changes in NKG2D expression in the parietal cortex in the four groups (Fig. 3a). Conversely,

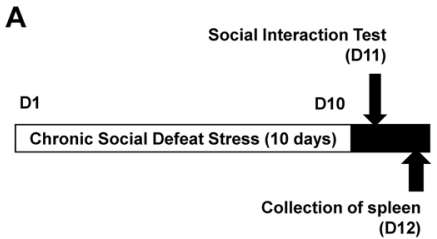

B

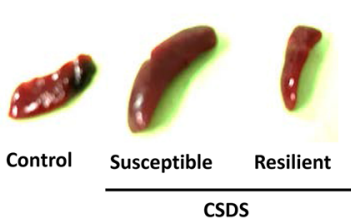

D
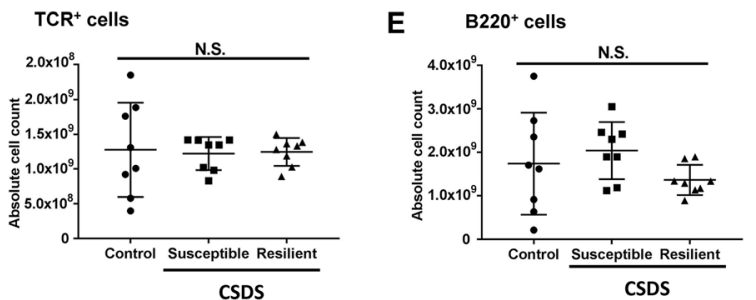

Fig. 1 Schedule of CSDS and FACS analysis. a The schedule of chronic social defeat stress (CSDS) model, social interaction test, and collection of spleen. CSDS was performed for 10 days (day 1- day 10). On day 11 , the social interaction test was performed to separate CSDS-susceptible mice and CSDS-resilient mice. Control (no CSDS) mice were used as control group. On day 12, spleen from the three groups was collected. b Representative picture of spleen from control (no CSDS) mice, CSDS-susceptible mice, and CSDS-resilient mice. Spleen weight of CSDS-susceptible mice was significantly higher than that of control mice and CSDS-resilient mice (one-way ANOVA, $F_{2,21}=437.136, P<0.001$ ). c Total cell number of spleen (one-way
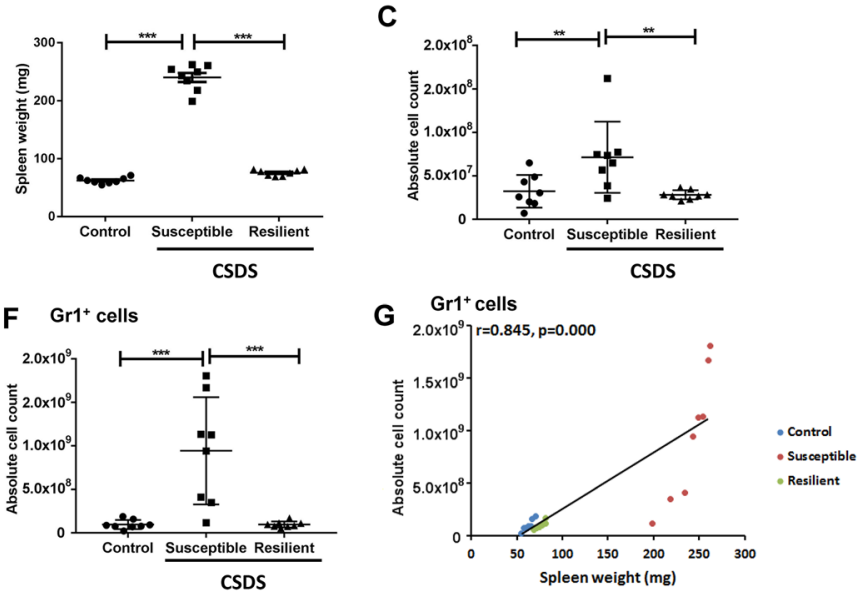

ANOVA, $\left.F_{2,21}=6.616, P=0.006\right)$. d T cells: The number of $\mathrm{TCR}^{+}$ cells in the spleen (one-way ANOVA, $F_{2,21}=0.032, P=0.968$ ). e B cells: The number of $\mathrm{B} 220^{+}$in the spleen (one-way ANOVA, $\left.F_{2,21}=1.429, P=0.262\right)$. f Granulocyte: The number of $\mathrm{Gr}^{+}$cells in the spleen (one-way ANOVA, $F_{2,21}=14.958, P<0.001$ ). $\mathrm{g}$ A positive correlation $(\mathrm{r}=0.845, P<0.001)$ between $\mathrm{Gr}^{+}$cells (for granulocytes) and spleen weight among three groups. Data are shown as mean \pm SEM. $(n=8)$. $* * P<0.01, * * * P<0.001$ ANOVA analysis of variance, $B 220$ B cells, $G r 1$ granulocyte receptor $1, C D 11 b$ a marker of macrophages, N.S. not significant, TCR T cell receptor 


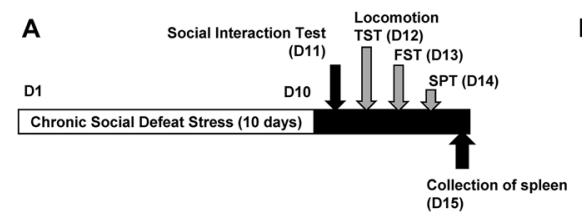

B

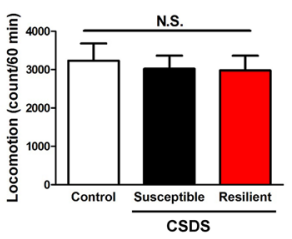

C

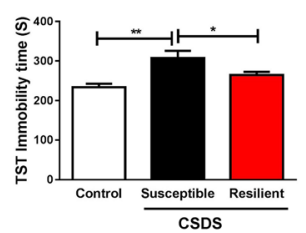

D

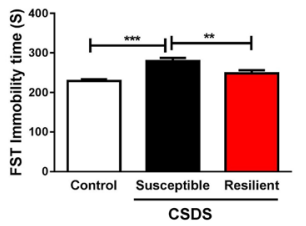

$\mathbf{E}$

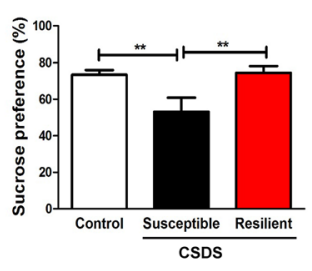

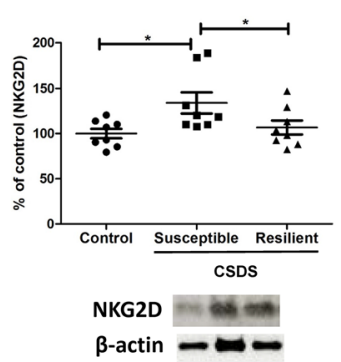

G

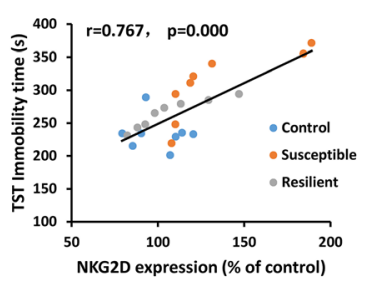

$\mathrm{H}$

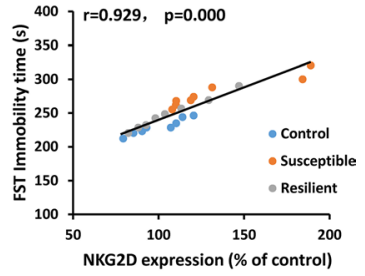

Fig. 2 Behavioral tests and NKG2D expression in the spleen of CSDS-susceptible mice and CSDS-resilient mice. a The schedule of CSDS model, social interaction test, behavioral tests, and collection of spleen. CSDS was performed for 10 days (day 1-day 10). On day 11, the social interaction test was performed to separate CSDS-susceptible mice and CSDS-resilient mice. On day 12, locomotion and TST were performed. On day 13, FST was performed. On day 14 , SPT was performed. On day 15 , spleen from the three groups was collected. Control (no CSDS) mice were used as control group. b Locomotion (one-way ANOVA, $F_{2,21}=0.118, P=0.889$ ). c TST (one-way ANOVA, $F_{2,21}=8.494, P=0.002$ ). d FST (one- way ANOVA, $F_{2,21}=13.317, P<0.001$ ). e SPT (one-way ANOVA, $\left.F_{2,21}=5.395, \quad P=0.013\right)$. f NKG2D expression in the spleen (one-way ANOVA, $F_{2,21}=4.278, P=0.028$ ). $g$ A positive correlation $(\mathrm{r}=0.767, \quad P<0.001)$ between TST immobility time and NKG2D expression in the spleen. $\mathbf{h}$ A positive correlation $(\mathrm{r}=0.929, P<0.001)$ between FST immobility time and NKG2D expression in the spleen. Data are shown as mean \pm SEM. $(n=8)$. $* P<0.05, * * P<0.01, * * * P<0.001$. ANOVA analysis of variance, $F S T$ forced swimming test, N.S. not significant, SPT sucrose preference test, TST tail suspension test

B

B Spleen

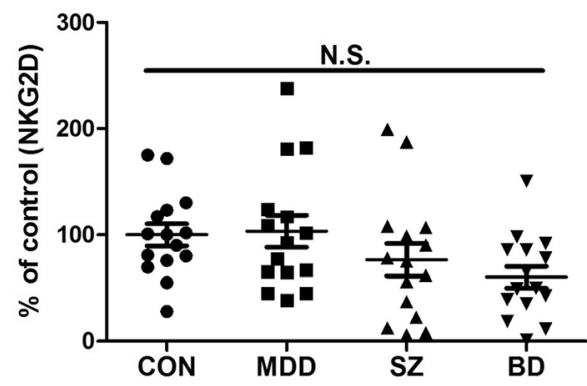

NKG2D

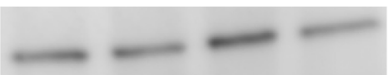

$\beta$-actin

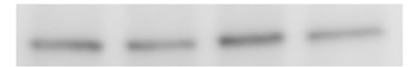

Fig. 3 Protein expression of NKG2D in the parietal cortex and spleen from patients with major psychiatric disorders. a Parietal cortex: There were no changes (one-way ANOVA, $F_{3,53}=3.004, P=0.051$ ) among the four groups. The bands of representative bands of Western blot analysis were shown. Data are shown as mean \pm SEM. $(n=15)$. b Spleen: There were significant changes (one-way ANOVA,

there were significant changes in NKG2D expression in the spleen in the four groups (Fig. 3b). Interestingly, NKG2D expression in the spleen was significantly higher in MDD
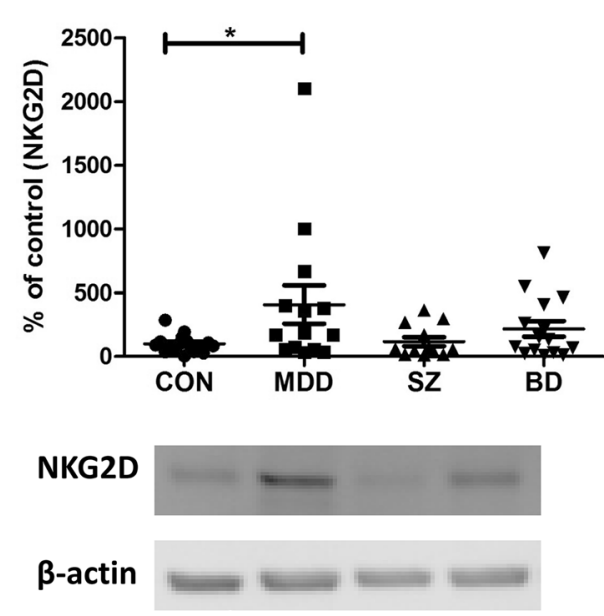

$\left.F_{3,52}=2.673, \quad P=0.048\right)$ among the four groups. Expression of NKG2D in the spleen of MDD patients was significantly $(P=0.012)$ higher than controls. ${ }^{*} P<0.05$. Data are shown as mean \pm SEM. $(n=12-15)$. The bands of representative bands of Western blot analysis were shown. $C O N$ control, $M D D$ major depressive disorder, $S Z$ schizophrenia, $B D$ bipolar disorder, N.S. not significant

patients than in controls (Fig. 3b). These findings indicate that increased NKG2D expression in the spleen of MDD patients might contribute to the pathogenesis of MDD. 


\section{Effects of (R)-ketamine in the splenic functions of CSDS-susceptible mice}

Previously, using rodent models of depression, we reported that $(R)$-ketamine has greater and longer-lasting antidepressant effects than does the US FDA-approved antidepressant $(S)$-ketamine [25-35]. Therefore, we examined whether $(R)$-ketamine $(10 \mathrm{mg} / \mathrm{kg})$ can ameliorate abnormal splenic functions in CSDS-susceptible mice (Fig. 4a). There were no changes in locomotion in the three groups (Fig. 4b). Compared to saline, $(R)$-ketamine significantly attenuated the increased immobility time of TST and FST in the CSDSsusceptible mice (Fig. 4c, d). Moreover, compared to saline, $(R)$-ketamine significantly enhanced the decreased sucrose preference of SPT in the CSDS-susceptible mice (Fig. 4e). Interestingly, a single injection of $(R)$-ketamine significantly ameliorated the increased size and weight of the spleen in CSDS-susceptible mice (Fig. 4f). Moreover, compared to saline, $(R)$-ketamine significantly attenuated the increased splenic NKG2D expression in CSDS-susceptible mice (Fig. 4g). Interestingly, in all groups, there were positive correlations between the immobility time of TST (or FST) and spleen weight (Figures S1A and S1B). Conversely, in all groups, there was a negative correlation between sucrose preference of SPT and spleen weight (Figure S1C). Moreover, in all groups, there was a positive correlation between splenic NKG2D expression and spleen weight (Figure S1D). These findings indicate that splenic NKG2D might contribute to depression-like phenotypes in CSDS-susceptible mice.

Subsequently, we conducted FACS analysis of spleen samples from the three groups. Compared to saline, $(R)$ ketamine $(10 \mathrm{mg} / \mathrm{kg}, 3$ days after injection) attenuated significantly the increased spleen cell number of CSDS-susceptible mice (Fig. 5a). Furthermore, compared to saline, $(R)$-ketamine attenuated significantly the increased number of NKG2D ${ }^{+}$cells in the spleen of CSDS-susceptible mice (Fig. 5b, c). There were also positive correlations between the immobility time of TST and FST (but not anhedonia) and splenic NKG2D expression in all groups (Figure S2A-S2C).

\section{Discussion}

The main findings of this study are as follows: first, in CSDS-susceptible mice, the spleen was larger and heavier than in both control (no CSDS) mice and CSDS-resilient mice. FACS analysis of spleen revealed that there were more granulocytes, but not $\mathrm{T}$ cells and $\mathrm{B}$ cells, in the CSDS-susceptible mice than in both control mice and CSDS-resilient mice. Interestingly, there were positive correlations between the number of granulocytes in the spleen and spleen weight. Thus, it appears that increased size and weight of the spleen
A
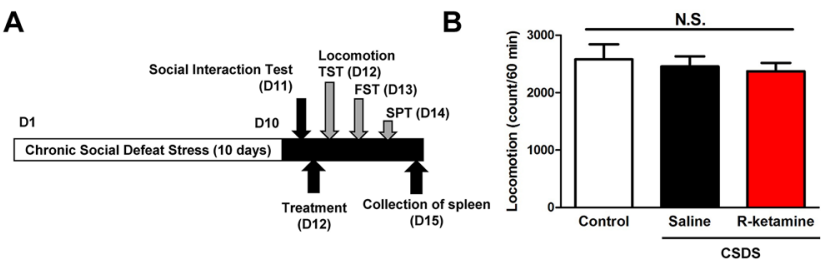

C

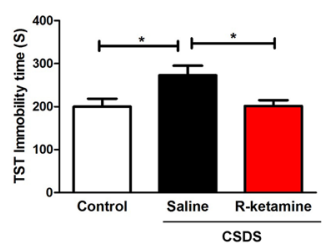

D

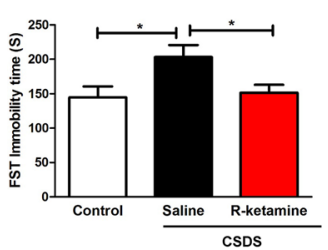

E

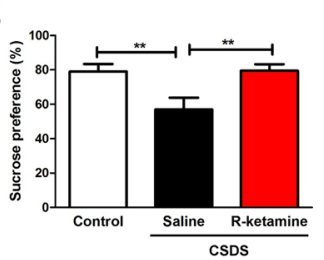

$\mathbf{F}$

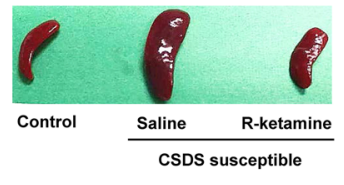

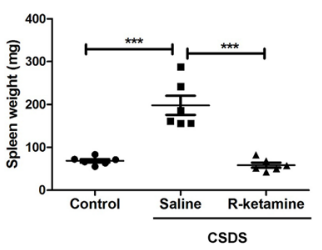

G

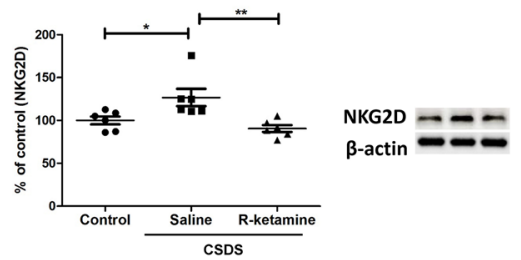

Fig. 4 Effects of $(R)$-ketamine on depression-like phenotypes, abnormal splenic functions in the CSDS-susceptible mice. a The schedule of CSDS model, social interaction test, treatment, behavioral tests, and collection of spleen. CSDS was performed for 10 days (day 1-day 10). On day 11, the social interaction test was performed to select CSDS-susceptible mice. Control (no CSDS) mice were used as control group. On day 12 , saline $(10 \mathrm{ml} / \mathrm{kg})$ or $(R)$-ketamine $(10 \mathrm{mg} / \mathrm{kg})$ was administered i.p. into CSDS-susceptible mice. Saline was administered i.p. into control (no CSDS) mice. Locomotion and TST were performed 1 and $3 \mathrm{~h}$ after injection, respectively. On day 13, FST was performed. On day 14, SPT was performed. On day 15, spleen from the three groups was collected. b Locomotion (one-way ANOVA, $F_{2,15}=0.270, P=0.767$ ). c TST (oneway ANOVA, $F_{2,15}=5.188, P=0.019$ ). d FST (one-way ANOVA,
$F_{2,15}=4.446, P=0.030$ ). e SPT (one-way ANOVA, $F_{2,15}=6.376$, $P=0.010)$. f Representative picture of spleen from saline-treated control (no CSDS) mice, saline-treated CSDS-susceptible mice, and $(R)$ ketamine-treated CSDS-susceptible mice. Spleen weight of $(R)$-ketamine-treated CSDS-susceptible mice was significantly lower higher than that of saline-treated CSDS-resilient mice (one-way ANOVA, $\left.F_{2,15}=12.052, P=0.001\right)$. $g$ Western blot analysis of NKG2D expression in the spleen (one-way ANOVA, $F_{2,15}=7.591, P=0.005$ ). The representative bands of Western blot analysis were shown. Data are shown as mean \pm SEM. $(n=6) . * P<0.05$, $* * P<0.01, * * * P<0.001$. ANOVA analysis of variance, ANOVA analysis of variance, FST forced swimming test, $N K G 2 D$ natural-killer receptor group 2, member D, N.S. not significant, $S P T$ sucrose preference test, $T S T$ tail suspension test 

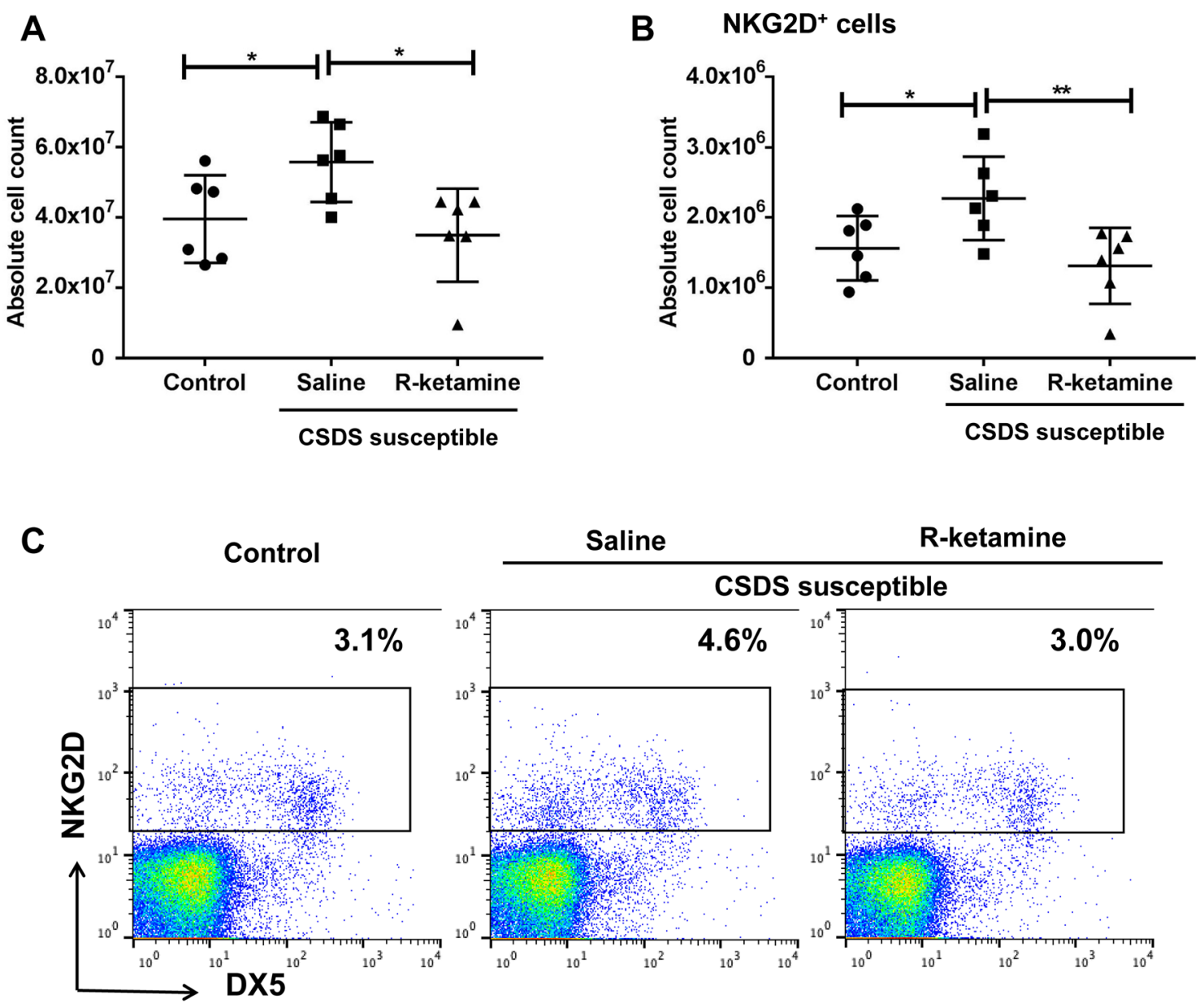

Fig. 5 FACS analysis of spleen samples of three groups. a Total cell number of spleen (one-way ANOVA, $F_{2,15}=4.659, P=0.027$ ). b The number of $\mathrm{NKG}_{2} \mathrm{D}^{+}$cells in the spleen (one-way ANOVA, $\left.F_{2,15}=5.207, \quad P=0.019\right)$. Data are shown as mean \pm SEM. $(n=6) . * P<0.05, * * P<0.01$. c FACS analysis of cells stained with antibodies against NKG2D and DX5 in spleen samples from saline-

in CSDS-susceptible mice might result from the increase in the number of granulocytes in the spleen of these mice. Second, NKG2D expression in the spleen of CSDS-susceptible mice was higher than that in both control mice and CSDSresilient mice. Interestingly, there were positive correlations between the immobility time of TST (or FST) and splenic NKG2D expression in the three groups. It appears that increased expression of NKG2D in the spleen may contribute to the depression-like phenotypes in CSDS-susceptible mice. Importantly, we found increased NKG2D expression in the spleen from MDD patients compared to controls, whereas there were no changes in the parietal cortex of MDD patients. Third, in the CSDS-susceptible mice, a single injection of $(R)$-ketamine had fast-acting antidepressant effects, consistent with our previous reports [29, 32-35]. Interestingly, three days after a single injection of $(R)$-ketamine, the increased size and weight of spleen in CSDS-susceptible treated control mice, saline-treated CSDS-susceptible mice, and $(R)$ ketamine-treated CSDS-susceptible mice. $(R)$-ketamine ameliorated the increased percentage of $\mathrm{NKG}_{2} \mathrm{D}^{+}$cells in the spleen of CSDSsusceptible mice. ANOVA analysis of variance, DX5 CD49b, NKG2D natural-killer receptor group 2, member D

mice recovered to control levels. Moreover, in these mice, there were correlations between the severity of depressionlike phenotypes and spleen weight. $(R)$-ketamine attenuated significantly the increased splenic NKG2D expression in the CSDS-susceptible mice. Moreover, $(R)$-ketamine attenuated significantly the increases in total cell number and NKG2D ${ }^{+}$ cells in the spleen of CSDS-susceptible mice. These findings indicate that splenic NKG2D confers resilience versus susceptibility in mice subjected to CSDS. Moreover, $(R)$ ketamine might, in part, exert antidepressant-like effects by normalizing splenic NKG2D in CSDS-susceptible mice. Finally, the brain-spleen axis may well, at least in part, play a role in stress-related psychiatric disorders (Fig. 6).

In 2018, McKim et al. [45] reported that the total number of cells within the spleen was increased $\sim 2.6$-fold after modified CSDS and that modified CSDS-induced numbers of erythrocytes, monocytes, and granulocytes within the 
Fig. 6 Proposed mechanisms of the role of brain-spleen axis in the stress-induced psychiatric disorders. Stress caused increase of spleen size and weight, resulting in abnormalities in spleen functions. Brain inflammation by stress might be mediated by brain-spleen axis (i.e., vagus nerve). NKG2D in spleen may play a role in the stress-related psychiatric disorders such as depression. Interestingly, $(R)$-ketamine ameliorates abnormalities of spleen functions and depressive symptoms by stress
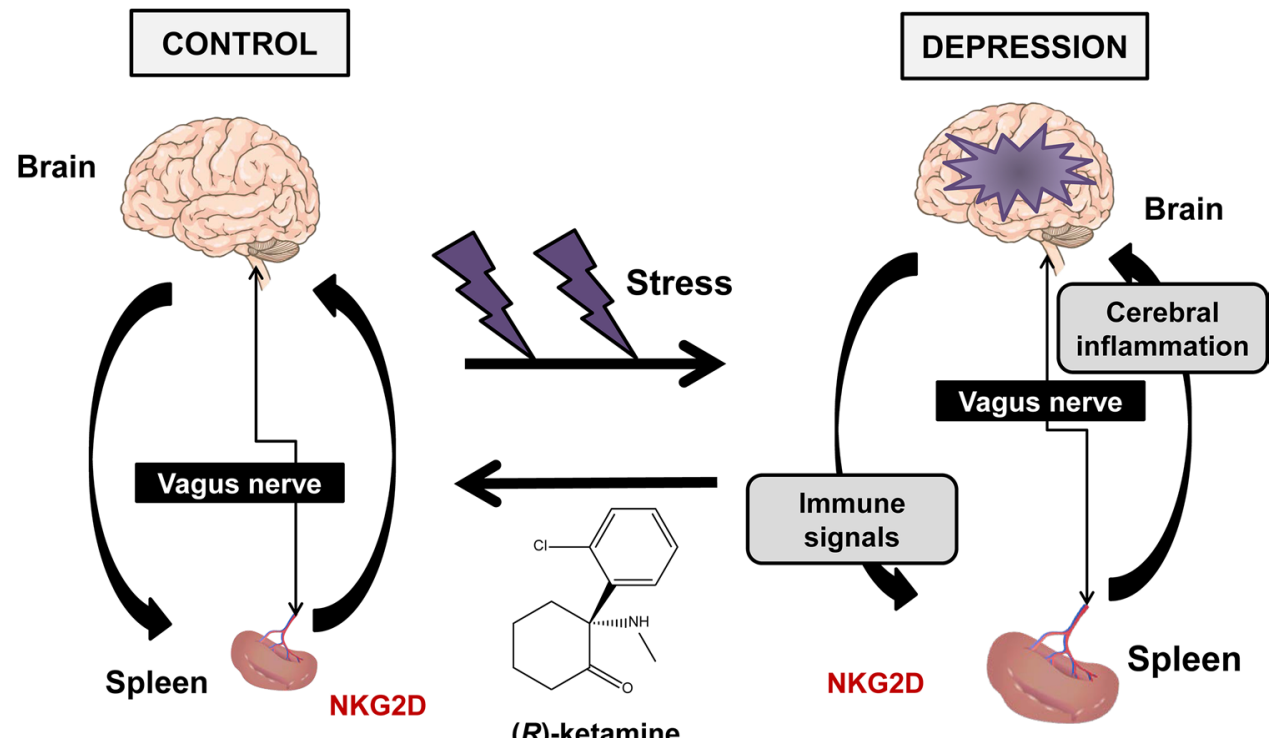

spleen are associated with $\mathrm{a} \sim$ twofold increase in spleen size and weight. However, they did not use a social interaction to divide susceptible mice and resilient mice, suggesting that they used both susceptible and resilient mice subjected to modified CSDS [45]. Notably, in the CSDS-resilient mice, spleen size and weight, in addition to splenic functions, were similar to those in control (no CSDS) mice. Collectively, it is likely that CSDS-induced alterations in splenic functions (i.e., granulocytes) confer stress susceptibility versus resilience in mice after CSDS, although there is a need for further detailed study on the spleen's role in stress resilience. Further, it is of great interest to study whether spleen size is changed in medication-free patients with stress-related disorders such as MDD.

NKG2D is a potent activator of the immune system that translates cellular stress into activation signals for immune cells [21, 44, 46]. Accumulating data support the role of inflammation- and stress-induced expression of NKG2D ligands in several immune-mediated diseases [21], indicating that NKG2D can be a therapeutic target for inflammation-related diseases. In this study, we found increased NKG2D expression in the spleen of CSDS-susceptible mice compared to both control and CSDS-resilient mice. We also found positive correlations between the immobility time of TST (or FST) and splenic NKG2D expression in control, susceptible, and resilient groups. It should be noted that the NKG2D expression in the spleen of CSDS-resilient mice was similar to that in control (no CSDS) mice. A study using NKG2D-deficient mice showed that there were fewer cells in the spleen of NKG2D-deficient mice than in the spleen of age-matched control mice, and that deficient mice were more resistant to murine cytomegalovirus infection [47]. Collectively, CSDS-induced increases in splenic NKG2D likely confer stress susceptibility versus resilience in mice after CSDS, although there is need for further study of the role of splenic NKG2D in stress resilience. Nonetheless, it is very interesting to study the role of splenic NKG2D in stress resilience versus susceptibility using spleen-specific NKG2D-deficient mice.

In this study, we also found positive correlations between the immobility time of TST (or FST) and NKG2D expression in the spleen in control, saline-treated susceptible, and $(R)$-ketamine-treated susceptible groups. Moreover, there was positive correlation between splenic NKG2D expression and spleen weight in the three groups, indicating that NKG2D may be involved in spleen function. Collectively, it is likely that increased splenic NKG2D expression may contribute both to the depression-like phenotypes and to increased spleen size and weight in CSDS-susceptible mice. It should be noted that a single injection of $(R)$-ketamine ameliorated the increased splenic NKG2D expression in CSDS-susceptible mice. It is of great interest to study whether $(R)$-ketamine can affect spleen size in patients with stress-related psychiatric disorders, as a clinical trial of $(R)$-ketamine is underway [26]. Additionally, $(R)$-ketamine might be a potential therapeutic drug for inflammationrelated diseases, as a clinical trial of anti-NKG2D antibody is underway for inflammation-related diseases such as Crohn's disease and rheumatoid arthritis [21, 48].

Multiple lines of evidence indicate that abnormal composition of gut microbiota may contribute to resilience versus susceptibility in rodents after either CSDS or inescapable electric stress [7, 49-54]. Further study of the role of braingut microbiota and spleen in stress susceptibility and resilience is also of interest (Fig. 6).

In conclusion, this study reveals that increased size and weight of the spleen in CSDS-susceptible mice might be associated with increases in the number of granulocytes 
in the spleen, and that splenic NKG2D might be associated with susceptibility versus resilience in mice following CSDS. Moreover, $(R)$-ketamine ameliorated abnormal splenic functions (i.e., increases in spleen size and NKG2D expression) in CSDS-susceptible mice. Finally, we propose a new hypothesis that the brain-spleen axis might, at least in part, play a role in stress-related psychiatric disorders.

Acknowledgements This study was supported by JSPS KAKENHI (to K.Z., 19K17054), AMED (to K.H., JP19dm0107119).

\section{Compliance with ethical standards}

Conflict of interest Dr. Hashimoto is an inventor on the filed patent on "The use of $(R)$-ketamine in the treatment of psychiatric diseases". Other authors declare no conflict of interest.

Open Access This article is licensed under a Creative Commons Attribution 4.0 International License, which permits use, sharing, adaptation, distribution and reproduction in any medium or format, as long as you give appropriate credit to the original author(s) and the source, provide a link to the Creative Commons licence, and indicate if changes were made. The images or other third party material in this article are included in the article's Creative Commons licence, unless indicated otherwise in a credit line to the material. If material is not included in the article's Creative Commons licence and your intended use is not permitted by statutory regulation or exceeds the permitted use, you will need to obtain permission directly from the copyright holder. To view a copy of this licence, visit http://creativecommons.org/licenses/by/4.0/.

\section{References}

1. McEwen BS (1998) Stress, adaptation, and disease. Allostasis and allostatic load. Ann N Y Acad Sci 840:33-44

2. Southwick SM, Vythilingam M, Charney DS (2005) The psychobiology of depression and resilience to stress: implications for prevention and treatment. Ann Rev Clin Psychol 1:255-291

3. Feder A, Nestler EJ, Charney DS (2009) Psychobiology and molecular genetics of resilience. Nat Rev Neurosci 10:446-457

4. Franklin TB, Saab BJ, Mansuy IM (2012) Neural mechanisms of stress resilience and vulnerability. Neuron 75:747-761

5. Russo SJ, Murrough JW, Han MH, Charney DS, Nestler EJ (2012) Neurobiology of resilience. Nat Neurosci 15:1475-1484

6. Dantzer R, Cohen S, Russo SJ, Dinan TG (2018) Resilience and immunity. Brain Behav Immun 74:28-42

7. Cathomas F, Murrough JW, Nestler EJ, Han MH, Russo SJ (2019) Neurobiology of resilience: interface between mind and body. Biol Psychiatry 86:410-420

8. Segerstrom SC, Miller GE (2004) Psychological stress and human immune system: a meta-analytic study of 30 years of inquiry. Psychol Bull 130:601-630

9. Chrousos GP (2009) Stress and disorders of the stress system. Nat Rev Endocrinol 5:374-381

10. Ménard C, Pfau ML, Hodes GE, Russo SJ (2016) Immune system and neuroendocrine mechanisms of stress vulnerability and resilience. Neuropsychopharmacology 42:62-80

11. Pfau ML, Russo SJ (2015) Peripheral and central mechanisms of stress resilience. Neurobiol Stress. 1:66-79

12. Mebius RE, Kraal G (2005) Structure and function of the spleen. Nat Rev Immunol 5:606-616
13. Bronte V, Pittet MJ (2013) The spleen in local and systemic regulation of immunity. Immunity 39:806-818

14. Lewis SM, Williams A, Eisenbarth SC (2019) Structure and function of the immune system in the spleen. Sci Immunol 4:eaau6085

15. Bailey MT, Engler H, Powell ND, Padgett DA, Sheridan JF (2007) Repeated social defeat increases the bactericidal activity of splenic macrophages through a Toll-like receptor-dependent pathway. Am J Physiol Regul Integr Comp Physiol 293:R1180-R1190

16. Powell ND, Bailey MT, Mays JW et al (2009) Repeated social defeat activates dendritic cells and enhances Toll-like receptor dependent cytokine secretion. Brain Behav Immun 23:225-231

17. Jiang W, Li Y, Sun J et al (2017) Spleen contributes to restraint stress induced changes in blood leukocytes distribution. Sci Rep 7:6501

18. González S, López-Soto A, Suarez-Alvarez B, López-Vazquez A, López-Larrea C (2008) NKG2D ligands: key targets of the immune response. Trends Immunol 29:397-403

19. Raulet DH, Gasser S, Gowen BG, Deng W, Jung H (2013) Regulation of ligands for the NKG2D activating receptor. Annu Rev Immunol 31:413-441

20. Zingoni A, Molfetta R, Fionda C et al (2018) NKG2D and its ligands: "One for all, all for one". Front Immunol 9:476

21. Babic M, Romagnani C (2018) The role of natural killer group 2 , member $\mathrm{D}$ in chronic inflammation and autoimmunity. Front Immunol 9:1219

22. Duman RS (2018) Ketamine and rapid-acting antidepressants: a new era in the battle against depression and suicide. F1000Res 7:659

23. Zanos P, Moaddel R, Morris PJ et al (2018) Ketamine and ketamine metabolites pharmacology: insights into therapeutic mechanisms. Pharmacol Rev 70:621-660

24. Krystal JH, Abdallah CG, Sanacora G, Charney D, Duman RS (2019) Ketamine: a paradigm shift for depression research and treatment. Neuron 101:774-778

25. Zhang K, Hashimoto K (2019) An update on ketamine and its two enantiomers as rapid-acting antidepressants. Expert Rev Neurother 19:83-92

26. Hashimoto K (2019) Rapid-acting antidepressant ketamine, its metabolites and other candidates: a historical overview and future perspective. Psychiatry Clin Neurosci 73:613-627

27. Yang C, Yang J, Luo A, Hashimoto K (2019) Molecular and cellular mechanisms underlying the antidepressant effects of ketamine enantiomers and its metabolites. Transl Psychiatry 9:280

28. Zhang JC, Li SX, Hashimoto K (2014) $R(-$-)-ketamine shows greater potency and longer lasting antidepressant effects than $S(+)$-ketamine. Pharmacol Biochem Behav 116:137-141

29. Yang C, Shirayama Y, Zhang JC, et al. (2015) $R$-ketamine: a rapidonset and sustained antidepressant without psychotomimetic side effects. Transl Psychiatry 5:e632

30. Hashimoto K (2016) $R$-ketamine: a rapid-onset and sustained antidepressant without risk of brain toxicity. Psychol Med 46:2449-2451

31. Hashimoto K (2016) Ketamine's antidepressant action: beyond NMDA receptor inhibition. Expert Opin Ther Targets 20:1389-1392

32. Yang C, Qu Y, Abe M, Nozawa D, Chaki S, Hashimoto K (2017) $(R)$-Ketamine shows greater potency and longer lasting antidepressant effects than its metabolite $(2 R, 6 R)$-hydroxynorketamine. Biol Psychiatry 82:e43-e44

33. Yang C, Qu Y, Fujita Y et al (2017) Possible role of the gut microbiota-brain axis in the antidepressant effects of $(R)$-ketamine in a social defeat stress model. Transl Psychiatry 7:1294

34. Yang C, Ren Q, Qu Y et al (2018) Mechanistic target of rapamycin-independent antidepressant effects of $(R)$-ketamine in a social defeat stress model. Biol Psychiatry 83:18-28 
35. Chang L, Zhang K, Pu Y et al (2019) Comparison of antidepressant and side effects in mice after intranasal administration of $(R$, $\mathrm{S})$-ketamine, (R)-ketamine, and (S)-ketamine. Pharmacol Biochem Behav 181:53-59

36. Golden SA, Covington HER 3rd, Berton O, Russo SJ (2011) A standardized protocol for repeated social defeat stress in mice. Nat Protocol 6:1183-1191

37. Zhang K, Hashimoto K (2019) Lack of opioid system in the antidepressant actions of ketamine. Biol Psychiatry 85:e25-e27

38. Zhang JC, Yao W, Dong C et al (2015) Comparison of ketamine, 7,8-dihydroxyflavone, and ANA-12 antidepressant effects in the social defeat stress model of depression. Psychopharmacology 232:4325-4335

39. Dong C, Zhang JC, Yao W et al (2017) Rapid and sustained antidepressant action of the mGlu2/3 receptor antagonist MGS0039 in the social defeat stress model: comparison with ketamine. Int $\mathbf{J}$ Neuropsychopharmacol 20:228-236

40. Torrey EF, Webster M, Knable M, Johnston N, Yolken RH (2000) The Stanley foundation brain collection and neuropathology consortium. Schizophrenia Res 44:151-155

41. Hashimoto K, Sawa A, Iyo M (2007) Increased levels of glutamate in brains from patients with mood disorders. Biol Psychiatry 62:1310-1316

42. Yang B, Ren Q, Zhang JC, Chen QX, Hashimoto K (2017) Altered expression of BDNF, BDNF pro-peptide and their precursor proBDNF in brain and liver tissues from psychiatric disorders: rethinking the brain-liver axis. Transl Psychiatry 7:e1128

43. Xiong Z, Zhang K, Ren Q, Chang L, Chen J, Hashimoto K (2019) Increased expression of inwardly rectifying Kir4.1 channel in the parietal cortex from patients with major depressive disorder. J Affect Disord 245:265-269

44. Jelenčić V, Lenartić M, Wensveen FM, Polić B (2017) NKG2D: a versatile player in the immune system. Immunol Lett 189:48-53
45. McKim DB, Yin W, Wang Y, Cole SW, Godbout JP, Sheridan JF (2018) Social stress mobilizes hematopoietic stem cells to establish persistent splenic myelopoiesis. Cell Rep 25:2552-2562

46. Lazarova M, Steine A (2019) The NKG2D axis: an emerging target in cancer immunotherapy. Expert Opin Ther Targets 23:281-294

47. Zafirova B, Mandarić S, Antulov R et al (2009) Altered NK cell development and enhanced NK cell-mediated resistance to mouse cytomegalovirus in NKG2D-deficient mice. Immunity 31:270-282

48. Vadstrup K, Bendtsen F (2017) Anti-NKG2D mAb: a new treatment for Crohn's disease? Int J Mol Sci 8:E1997

49. Yang C, Fujita Y, Ren Q, Ma M, Dong C, Hashimoto K (2017) Bifidobacterium in the gut microbiota confer resilience to chronic social defeat stress in mice. Sci Rep 7:45942

50. Szyszkowicz JK, Wong A, Anisman H, Merali Z, Audet MC (2017) Implications of the gut microbiota in vulnerability to the social avoidance effects of chronic social defeat in male mice. Brain Behav Immun 66:45-55

51. Kentner AC, Cryan JF, Brummelte S (2019) Resilience priming: Translational models for understanding resiliency and adaptation to early life adversity. Dev Psychobiol 61:350-375

52. Gururajan A, van de Wouw M, Boehme M et al (2019) Resilience to chronic stress is associated with specific neurobiological, neuroendocrine and immune responses. Brain Behav Immun 80:583-594

53. Zhang K, Fujita Y, Chang L et al (2019) Abnormal composition of gut microbiota is associated with resilience versus susceptibility to inescapable electric stress. Transl Psychiatry 9:231

54. Wang S, Qu Y, Chang L, Pu Y, Zhang K, Hashimoto K (2020) Antibiotic-induced microbiome depletion is associated with resilience in mice after chronic social defeat stress. J Affect Disord 260:448-457 